\title{
Research on Regional Development of Rail Transit -- Taking Guangfo Metro as an example
}

\author{
Pan Tang ${ }^{1}$ \\ ${ }^{1}$ Wuhan University of Technology, School of Management Wuhan, China
}

\begin{abstract}
The shortest path model is selected to evaluate the regional traffic accessibility. Three index factors, gross regional product, total retail sales of consumer goods, and the number of permanent residents in the region, were selected, and the weight sum was given by entropy weight method to evaluate the regional economic intensity. The gravity model adding the regional accessibility index considering traffic factors is selected to evaluate the regional linkages. By integrating the three indexes, an evaluation index system of rail transit benefits is formed to study the utility of rail transit to the economic development of surrounding areas, and to provide scientific theoretical basis and guidance for the government to carry out urban construction and rail transit management.
\end{abstract}

\section{Introduction}

With the development of economy, traffic problems like traffic congestion are constantly emerging in various cities, while rail transit, with its advantages such as fast speed, large volume and good safety performance, satisfies huge traffic demand well, and is gradually recognized by major cities at home and abroad. Moreover, as the backbone of urban transportation system, rail transit can not only relieve the increasingly crowded road traffic in big cities, but also provide support for urban planning and construction, inject vitality into urban economic chain from the source, and improve the comprehensive value of the city. Accordingly, with the acceleration of urbanization, China is also rising a wave of rail transit construction. But can we break the bottleneck of regional economic development through the construction of rail transit under any circumstances so as to achieve the purpose of promoting regional economic development? In urban construction, how should rail transit construction be planned?

Based on the analysis of predecessors, this paper, by using traffic accessibility, regional linkages and the regional economic theory, discusses the rail transit on surrounding the development of the regional economic ties and utility, and hope to provide the government scientific theoretical basis and guidance to track traffic management, make the city traffic plan and management work scientific and predictable, and have important theoretical significance and application value.

\section{2 literature review}

Through literature analysis, it can be known that Zhang Li, Zhu Changning and Cao Lina found the interaction between regional accessibility and regional advantage by using the favorable average travel time model and other models to research the Shanghai-Nanjing Intercity highspeed railway 4. Xiang Qiannan and Chen Yihua improved the model of regional economic relations by adding the indicators of regional accessibility and accessibility resistance, and found the promoting effect of rail transit on relatively marginal areas by combining with the example of chongqing rail transit 1. Zhang Zhixue used the research methods of GIS network analysis, qualitative analysis and quantitative evaluation, combined with regional connection, and found the relationship between accessibility and the interactive development of regional economy 7. Li Peiquan and Cao Xiaoshu used the gravity measurement model, network connectivity and the shortest distance measurement model to find out the intensity of economic connection and spatial pattern of accessibility in Guangzhou - Foshan metropolis circle 6.

Taking what has been discussed into consideration, we can find that most of the predecessors have studied the relationship between traffic accessibility and regional linkages or regional economic development, but ignored the important relationship among regional accessibility, regional linkages and regional economy, which is crucial to regional development. With the acceleration of urban urbanization in China, the importance of urban rail transit has become increasingly prominent. Therefore, it is necessary to make an in-depth investigation of the relationship between the three to provide suggestions for urban planning and management in China. 


\section{Model building}

\subsection{Traffic accessibility}

Traffic accessibility is an effective index to measure network traffic structure, and the level of traffic accessibility is a powerful reflection of regional traffic level. Currently, there are four types of models to evaluate regional accessibility: shortest path model, economic potential model, favorable average travel time model and daily accessibility model. Using the shortest path model for calculation, the accessibility index $A_{i}$ of region $\mathrm{i}$ is defined as the mean value of the sum of the shortest travel time from region $i$ to other regions $\mathrm{j}(\mathrm{i} \neq \mathrm{j})$, and here follows the formula:

$$
A_{i}=\frac{1}{n} \cdot \sum_{j=1, j \neq i}^{n} T_{i j}
$$

The smaller the value of $A_{i}$ is, the better the traffic accessibility is.

\subsection{Economic intensity}

In order to make regional economic intensity more representative, the following factors that can comprehensively reflect regional economic intensity are selected for analysis: $X_{1}$ is regional GDP, reflecting the level of regional economic development; $X_{2}$ is the total retail sales of social consumer goods, representing the purchasing power of regional residents. If the purchasing power is strong, the living standard of the residents will be relatively high, and except meeting basic needs, they will have enough money to enjoy life. $X_{3}$ is the population of the whole region, which directly reflects the overall size of the region. The above three indicators are all positive indicators, that is, the stronger the regional economic strength is, the greater the index value is.

Dimensionless processing is carried out, in order to eliminate the influence of dimensionality of individual index factors. Considering that the relative importance of different index factors is different, the entropy weight method is used to assign the index factors. According to the explanation of the basic principles of information theory, the smaller the information entropy of the index, the larger the information provided by the index. Which means when it comes to the regional economic strength, the greater the role of indicator factors, the higher it weights. The process is as follows:

For an evaluation problem with m evaluation indexes and $\mathrm{n}$ objects, the following evaluation matrix is established:

$$
\mathrm{R}^{\prime}=\left|\begin{array}{ccc}
r^{\prime}{ }_{11} & \ldots & r^{\prime}{ }_{1 n} \\
\ldots & \cdot & \ldots \\
r^{\prime}{ }_{m 1} & \ldots & r^{\prime}{ }_{m n}
\end{array}\right|
$$

Standardizing formula (2) comes the result: $R=\left(r_{i j}\right)_{m n}$

Among them:

$$
r_{i j}=\left(r_{i j}^{\prime}-\min \cdot r_{i j}^{\prime}\right) /\left(\max \cdot r_{i j}^{\prime}-\min r_{i j}^{\prime}\right)
$$

For research purposes, some concepts are defined as follows.

(1) Evaluation of the entropy of the index. In the evaluation problem of $m$ indexes and $n$ objects, the entropy of the ith index is defined as:

$$
H_{i}=-K \sum_{j=1}^{n} f_{i j} \ln f_{i j}
$$

Among them: $f_{i j}=r_{i j} / \sum_{j=1}^{n} r_{i j} ; \mathrm{K}=1 / \mathrm{ln} \cdot \mathrm{n}$.

(If $f_{i j}=0, f_{i j} \ln f_{i j}=0$ )

(2) The entropy weight of the index. In the evaluation problem of $m$ indexes and $n$ objects, the entropy weight of the ith index is defined as:

$$
W_{i}=\left(1-H_{i}\right) /\left(m-\sum_{j=1}^{n} H_{i}\right)
$$

Among them: $\sum_{j=1}^{n} W_{i}=1,0 \leq W_{i} \leq 1$.

$W_{i}$ is the weight of regional economic intensity index factor, including regional GDP, total retail sales of social consumer goods, and the population of the whole region.

\subsection{Regional linkages}

There are many indicators to measure the interregional relationship, among which the gravity measurement model is one of the most widely used models in recent years. It borrows the idea of gravity model in physics and believes that the spatial effect of geographical entities such as cities decreases with the increase of spatial distance. It comprehensively considers the location of the entity in the traffic network and the distribution mode of other entities in the network. The formula is as follows:

$$
\begin{gathered}
R_{i j}=\sqrt{P_{i} V_{i}} \cdot \sqrt{P_{j} V_{j}} / D_{i j} \\
R_{i}=\sum_{j=1}^{n} R_{i j}
\end{gathered}
$$

Among them, $P_{i}$ and $V_{i}$ represents the regional population and regional GDP of region i respectively $P_{j}$ and $V_{j}$ represents the regional population and regional GDP of region $\mathrm{j}, D_{i j}$ represents the shortest distance between region $\mathrm{i}$ and region $\mathrm{j} ; R_{i j}$ represents the regional connection strength between region $\mathrm{i}$ and region $\mathrm{j} ; R_{i}$ represents the overall economic linkages degree of region $\mathrm{i}$ in the network; the larger the value is, the stronger the overall economic linkages degree of region $i$ in the network is.

In the model, $D_{i j}$, represented by the distance between regions, cannot reflect the influence of regional traffic factors, while regional connection and regional traffic accessibility have a mutual promoting cycle, so $A_{i j}$, an indicator of regional accessibility, is adopted to replace $D_{i j}$. Rewrite equation (6) and (7) as follows: 


$$
\begin{gathered}
R_{i j}=\sqrt{P_{i} V_{i}} \cdot \sqrt{P_{j} V_{j}} / A_{i j} \\
R_{i}=\sum_{j=1}^{n} R_{i j}
\end{gathered}
$$

$R_{i}$ is the overall economic linkage degree of region $\mathrm{i}$ in the network which is the regional connection index value in this paper.

\section{Data acquisition and processing}

\subsection{Area selection}

As an important means of transportation connecting the two cities, Guangzhou-Foshan rail transit has been developing continuously, promoting the connection between regions and bringing development to regional economy. As one of the important deployment strategies of Guangzhou-Foshan integration construction and Guangzhou-Foshan integration, Guangfo Metro plays an irreplaceable role in the political, economic and cultural exchanges between the two cities. Therefore, this paper chooses Guangfo Metro and its influence area as the research object. The Guangfo Metro was officially opened in November 2010, and by December 2018, the post-phase 1 section has been put into trial operation. Thus, the data of 2010 and 2018 are selected as the data before and after the opening of Guangfo Metro, and the eight areas around the metro, Liwan District, Haizhu District, Chancheng District, Panyu District, Yuexiu District, Tianhe District, Nanhai District and Shunde District, are selected as the objects for analysis and research.

\subsection{Data acquisition}

ArcGIS can be used to respectively calculate the shortest travel time between regions in 2010 and 2018, and then according to The Statistical Yearbook of Guangzhou and Foshan, relevant data of regional GDP, total retail sales of social consumer goods and regional population of each region can be obtained.

\section{Empirical analysis}

\section{1 traffic accessibility}

As can be seen from Figure 1, in 2010, haizhu District, Liwan District and Yuexiu District had the highest accessibility, which was 26.4, 26.7 and 27.3, respectively. The three districts, located in the central area of Guangzhou, owned strong economic strength and relatively mature traffic network, which makes its accessibility higher. Shunde District comes the lowest, the accessibility reached 44.7, which was 18.3 higher than Haizhu District. After the opening of Guangfo Metro in 2018 , it is obvious that the accessibility of all districts has been improved, indicating that rail transit can indeed improve the accessibility of regional traffic. Nanhai District showed the biggest change, with an decrease of
3.19. Nanhai District is a marginal area, relatively far from other areas, and there is no direct route to some areas, so sometimes detour is needed. Rail transit solves such problems well, so the change of accessibility is relatively significant. However, the accessibility of Shunde District and Panyu District had little change, and the traffic accessibility still had some room for improvement.

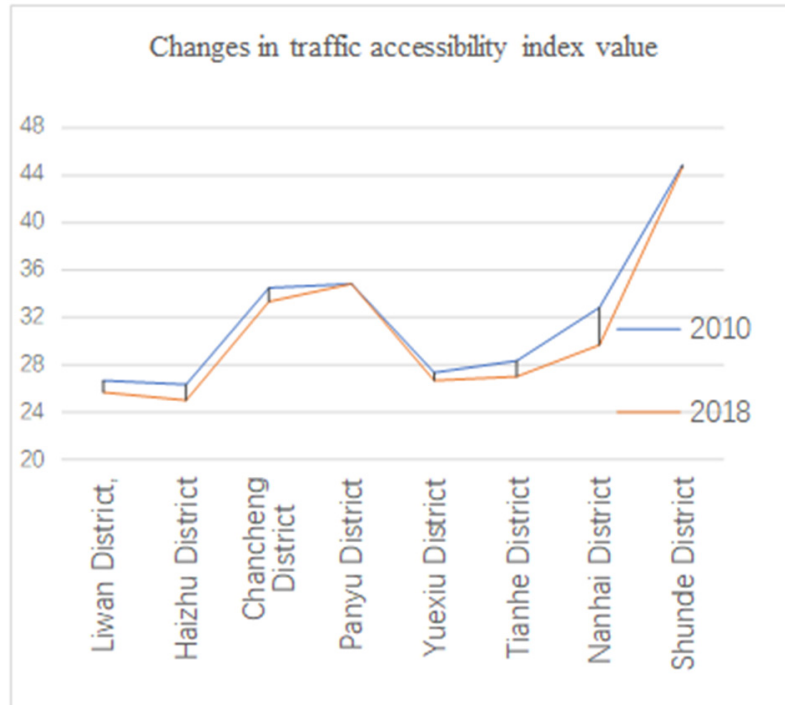

Fig 1. Changes of regional traffic accessibility index value in 2010 and 2018.

\subsection{Economic intensity}

By using formulas (2) to (5), the regional economic strength index factor data in 2010 are processed, and the evaluation matrix is obtained as follows:

$$
\mathrm{R}^{\prime}=\left|\begin{array}{ccc}
r_{11}^{\prime} & \ldots & r_{1 n}^{\prime} \\
\ldots & \cdot & \ldots \\
r_{m 1}^{\prime} & \ldots & r_{m n}^{\prime}
\end{array}\right|
$$

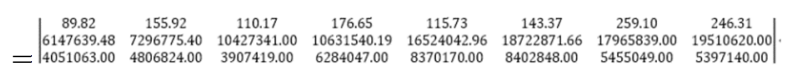

By standardizing $\mathrm{R}^{\prime}$, the following results can be obtained:

$\mathrm{R}^{\prime}=\left|\begin{array}{llllllll}0.00 & 0.39 & 0.12 & 0.51 & 0.15 & 0.32 & 1.00 & 0.92 \\ 0.00 & 0.09 & 0.32 & 0.34 & 0.78 & 0.94 & 0.88 & 1.00 \\ 0.03 & 0.06 & 0.00 & 0.53 & 0.99 & 1.00 & 0.34 & 0.33\end{array}\right|$

Then comes: $H_{1}=0.83, H_{2}=0.85, H_{3}=0.80$.

Similarly, the evaluation matrix $\mathrm{R}^{\prime}$ in 2018 is obtained as follows:

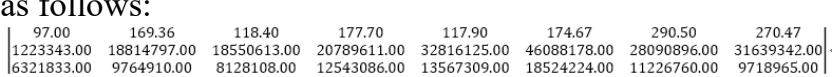

Standardized the R' and after further process comes $H_{1}$ $=0.82, H_{2}=0.86$, and $H_{3}=0.86$.

Thus, the factor weight $W_{i}$ in 2010 and 2018 can be obtained respectively, as shown in Table 1 . According to $W_{i}$, the economic strength of the 8 regions in 2010 and 2018 can be calculated respectively, as shown in Figure 2.

It can be seen that since the opening of Guangfo Metro, the regional economy has made great progress, and the economic intensity has been significantly improved. Before the opening, the average economic strength of each region was 6085428.68 , but after the opening, the average rose to 11416796.40 , with an increase of $88 \%$. The opening of subway improves the accessibility of each region, improves the transportation conditions for regional 
external contact, reduces transportation costs, strengthens commodity circulation and increases regional foreign exchanges, which is conducive to the expansion of the original market and the formation of new markets, thus promoting the development of regional economy. Among them, the economic intensity of Tianhe District increased the most, which was $130 \%$. Maybe because of the low accessibility before the opening of the subway limited the economic development of the region; the opening of the subway reduced the limit of accessibility, so the economy developed greatly. The economic intensity growth of Chancheng District is not high, and its traffic accessibility is also at a low level, which may be because the low level of traffic accessibility limits its economic growth. The accessibility of Liwan District is relatively high, but the change of regional economic intensity is relatively low, which may be because the main factor restricting its economic development is not traffic accessibility.

Table1. The weight of economic intensity index factor in

\begin{tabular}{|c|c|c|c|}
\hline \multicolumn{4}{|c|}{2010 and 2018} \\
\hline $\mathbf{2 0 1 0}$ & 0.33 & 0.29 & $\boldsymbol{W}_{\mathbf{3}}$ \\
\hline $\mathbf{2 0 1 8}$ & 0.39 & 0.31 & 0.38 \\
\hline
\end{tabular}

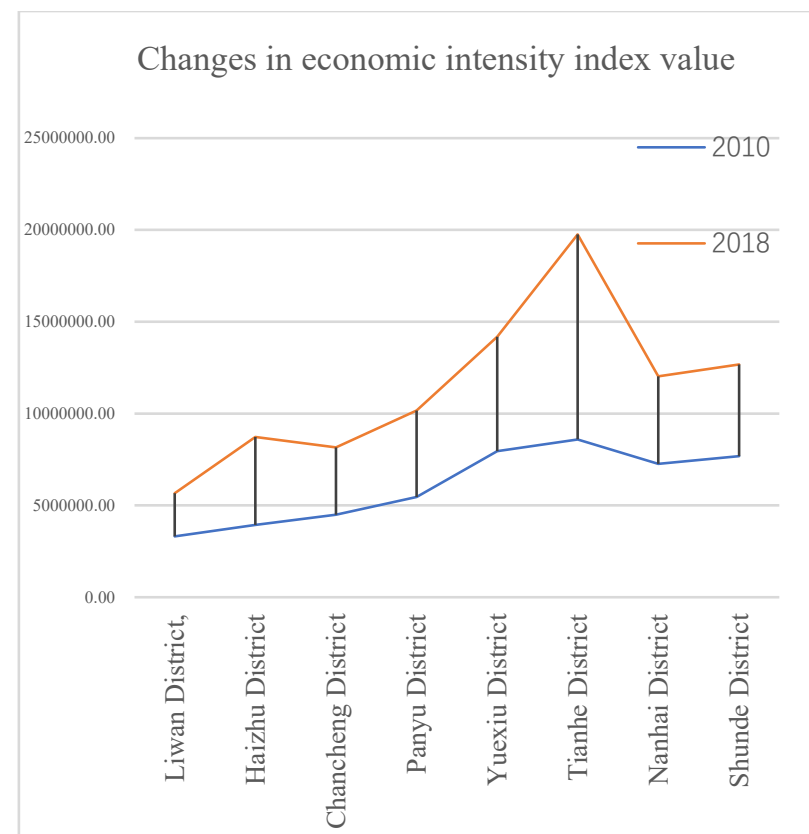

Fig 2. Changes of regional economic intensity index value in 2010 and 2018 .

\subsection{Regional linkages}

Before the opening of the subway, connections between different regions were at a relatively low level, with Panyu and Shunde Districts as the lowest. It may be because of the low traffic accessibility, poor transportation conditions, long transportation distance and too much time spent in the region, so the transportation cost is high, which is not conducive to reaching the further region. The opening of the subway greatly improved the regional traffic accessibility, improved the transportation conditions of the external connections between regions, and strengthened the inter-regional connections, with an increase of $121 \%$.
For enterprises, it is easier for their products to reach new markets or places of origin with lower operating costs, and their profits have been relatively increased. And for the region, it stimulated the regional economy, then regional economic level was improved. You can find the result in figures 1 through 3 . At the same time, it can be found that traffic accessibility, regional linkages, regional economic intensity change it the same direction. After the opening of the subway, the accessibility of Tianhe District, Nanhai District and Haizhu District was improved a lot, the interregional contact was more frequent, the economy was well developed, and the economic intensity was greatly improved.

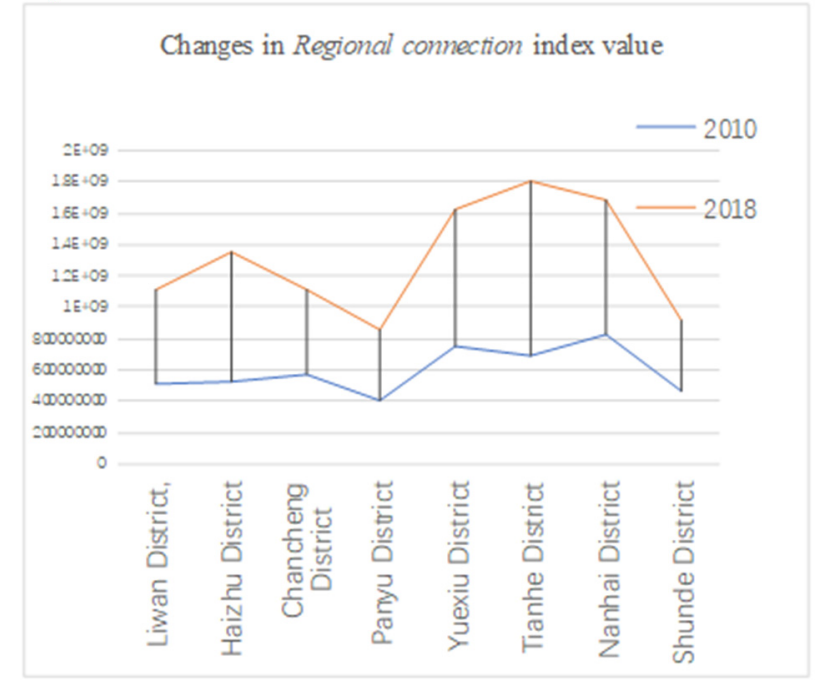

Fig 3. Changes of Regional linkages degree index value in 2010 and 2018.

\section{Conclusions and Recommendations}

\subsection{Conclusions}

\subsubsection{Regional effect of rail transit on traffic accessibility improvement.}

The marginal areas are far away from other areas, with sparse road network and poor traffic accessibility. The construction of rail transit has enhanced the density of road network and solved such problems well, so it has a significant impact on marginal areas. In the central area, the original road network is relatively dense with high traffic accessibility. The construction of rail transit may mainly play a role of traffic diversion, so the change of accessibility is small.

\subsubsection{Interaction between traffic accessibility and regional economy.}

In areas with low accessibility, the construction of rail transit has broken through the limitation of accessibility, strengthened regional external relations, and accelerated economic development. However, the situation is different for regions with high accessibility. At this time, we should take the advantages of high accessibility, and pay more attention to regional economic development, while the 
status of accessibility should not be ignored. Because the improvement of accessibility will promote the development of industries along the transportation line, and gradually form scale effect, so as to promote the development of regional economy. With the improvement of regional economic level, it can also stimulate the generation of transport demand, promote the further development of traffic construction, improve the accessibility level, form a good interactive cycle with regional economic development, and promote the coordinated development of the region.

\subsection{Positive relationship among traffic accessibility, regional linkages and regional economic development.}

The development resources of a region are limited, but by strengthening the construction of transportation, we can improve the regional accessibility, the regional linkages, and gragually stimulate the accumulation effect of regional economy, so as to make a better use of the resource advantages of each region and promote the further development of regional economy.

\subsection{Recommendations}

\subsubsection{Pay attention to the construction of rail transit when carrying out urban planning and management.}

As a subsystem of traffic planning, rail transit can not only relieve the traffic congestion; it can also active area economy, pull area to develop. In short, it is of great significance to regional development.

\subsubsection{Carring out the investment and construction of rail transit reasonablely according to the characteristics of different stages of regional economic development.}

Excessive investment and construction of rail transit is likely to produce a chain reaction of idle and waste of resources, while too low investment and construction of rail transit may lead to the suppression of regional economic development.

\subsubsection{More factors affecting the coordination with urban planning should be considered.}

We should strengthen the coordination between urban plannings, give full play to the good interaction between rail transit construction and urban planning, and promote the high-quality and sustainable development of China's urban modernization.

\section{Acknowledgement}

Financially supported by self-determined and innovative research funds of WUT

\section{References}

1. Xiang Qiannan, Chen Yihua. Research on the accessibility of rail transit and regional economic development $[\mathrm{J}]$. Railway transportation and economy, 2010,32(11):69-72.

2. Zhang Xianzhi. Research on the Influence of Rail Transit on regional Economic Development [D]. Chongqing University, 2007.

3. Li Ziwei, Hu Kai. Research on the Dynamic Relationship of transportation Infrastructure based on accessibility to industrial development in Gansu Province [J]. Value Engineering, 2020,39(01):67-72.

4. Zhang Li, Zhu Changning, Cao Lina. Research on the influence of shanghai-nanjing intercity high-speed railway on regional accessibility $[\mathrm{J}]$. Railway transport and economy, 2013,35(01):82-87.

5. Chen Mengxiao. Economic spatial Connection and Evolution Trend of National Urban Agglomerations - A Case study of Ha-Chang Urban agglomerations [J] Economic geography, 2020,40(05):99-105.

6. Li Peiquan, Cao Xiaoshu. Accessibility and spatial pattern of road network in guangfo metropolitan area [J]. Economic geography, 2011,31(03):371-378.

7. Zhang Zhixue. Research on the Evolution of Regional Accessibility and its Relationship with Regional Economic Development [D]. Northwestern University, 2009.

8. Liu Hailong, Bao Anming, Chen Xi, Zhang Xiaolei, Zhang Jinying. Analysis on the Influence of Traffic Accessibility on Regional Economy in Xinjiang [J]. Chinese Journal of Geography, 2008(04):428-436. 